\title{
Expression of Angiogenesis Regulatory Proteins and Epithelial-Mesenchymal Transition Factors in Platelets of the Breast Cancer Patients
}

\author{
Hui Han, ${ }^{1,2}$ Fang-Li Cao, ${ }^{2}$ Bao-Zhong Wang, ${ }^{2}$ Xue-Ru Mu, ${ }^{3}$ \\ Guang-Yao Li, ${ }^{4}$ and Xiu-Wen Wang ${ }^{1}$ \\ ${ }^{1}$ Department of Oncology, Qilu Hospital, School of Medicine, Shandong University, No. 107 Wenhua Xi Road, Jinan, \\ Shandong 250012, China \\ ${ }^{2}$ Department of Oncology, Liaocheng People's Hospital and Liaocheng Clinical School of Taishan Medical University, \\ Liaocheng 252000, China \\ ${ }^{3}$ Department of Oncology, Shandong Province-Owned Hospital, Shandong University, Jinan 250012, China \\ ${ }^{4}$ Department of Hematology, Liaocheng People's Hospital, Medical School of Liaocheng, Taishan Medical University, \\ 67 Dong Chang Xi Lu, Liaocheng, Shandong 252000, China
}

Correspondence should be addressed to Xiu-Wen Wang; xiuwenwang12@sdu.edu.cn

Received 21 February 2014; Accepted 14 August 2014; Published 14 October 2014

Academic Editor: Jahn M. Nesland

Copyright (C) 2014 Hui Han et al. This is an open access article distributed under the Creative Commons Attribution License, which permits unrestricted use, distribution, and reproduction in any medium, provided the original work is properly cited.

Platelets play a role in tumor angiogenesis and growth and are the main transporters of several angiogenesis regulators. Here, we aimed to determine the levels of angiogenesis regulators and epithelial-mesenchymal transition factors sequestered by circulating platelets in breast cancer patients and age-matched healthy controls. Platelet pellets (PP) and platelet-poor plasma (PPP) were collected by routine protocols. Vascular endothelial growth factor (VEGF), platelet-derived growth factor BB (PDGF-BB), thrombospondin-1 (TSP-1), platelet factor 4 (PF4), and transforming growth factor- $\beta 1$ (TGF- $\beta 1$ ) were measured by enzyme-linked immunosorbent assay. Angiogenesis-associated expression of VEGF $\left(2.1 \mathrm{pg} / 10^{6}\right.$ platelets versus $0.9 \mathrm{pg} / 10^{6}$ platelets, $\left.P<0.001\right)$, PF4 $\left(21.2 \mathrm{ng} / 10^{6}\right.$ platelets versus $10.2 \mathrm{ng} / 10^{6}$ platelets, $\left.P<0.001\right)$, PDGF-BB $\left(42.9 \mathrm{pg} / 10^{6}\right.$ platelets versus $19.1 \mathrm{pg} / 10^{6}$ platelets, $\left.P<0.001\right)$, and TGF- $\beta 1\left(15.3 \mathrm{ng} / 10^{6}\right.$ platelets versus $4.3 \mathrm{ng} / 10^{6}$ platelets, $\left.P<0.001\right)$ differed in the PP samples of cancer and control subjects. In addition, protein concentrations were associated with clinical characteristics $(P<0.05)$. Circulating platelets in breast cancer sequester higher levels of PF4, VEGF, PDGF-BB, and TGF- $\beta 1$, suggesting a possible target for early diagnosis. VEGF, PDGF, and TGF- $\beta 1$ concentrations in platelets may be associated with prognosis.

\section{Introduction}

Breast cancer is a common malignancy in women and has a high mortality rate [1]. There are no predictive markers for metastasis or prognosis, although more attention has been paid to early detection of breast cancer metastasis.

The oncology community has long been interested in biological markers for tumor surveillance, early recurrence, and therapeutic efficacy. Tumor growth and metastasis depend on angiogenesis $[2,3]$ and angiogenesis regulators are considered ideal diagnostic markers and therapeutic targets. Studies have shown that monitoring levels of angiogenesis markers in biological fluids can be used to assess tumor growth and development [4-6] but they do not facilitate early diagnosis $[7,8]$. There are studies showing that platelets play an important role in tumor angiogenesis and growth [9-14]. There are many regulatory proteins and proangiogenic endothelial cell growth factors in platelets, such as VEGF, PDGF, PF4, TSP-1, TGF- $\beta$, and bFGF [15-17]. Most of these proteins are released by platelets directly into the tumor microenvironment [18]. Studies of early phase for tumor formation in mice have shown increased levels of angiogenic proteins in platelets; however, there is no corresponding change in plasma [19]. It has been reported that VEGF was expressed in cancer patients' platelets [20]. However, it is unclear whether coexpression of multiple angiogenesis regulators in platelets is 
associated with breast cancer progress. In the present study, there is less study about relationship between these proteins and breast cancer prognosis.

In the present study, we aim to explore whether the increased protein levels in circulating platelets are associated with breast cancer progression and if these proteins can be biomarkers for breast cancer diagnosis. Therefore, we measured VEGF, TSP-1, PF4, and PDGF as well as analyzed the epithelial-mesenchymal transition factor TGF- $\beta 1$ in breast cancer patients and healthy controls. In addition, we investigated the association between these indicators and clinical characteristics.

\section{Materials and Methods}

2.1. Study Population. Breast cancer samples at different tumor stages were collected from patients at the Surgery and Oncology Departments at Liaocheng People's Hospital $(n=$ 37). Patients with platelet disorders or a medication history that would affect platelet physiology were excluded. Samples from the Department of General Surgery were collected before the operations. Samples from the Department of Oncology were collected at least 3 months after the conclusion of chemotherapy, radiotherapy, or medications for thrombocytopenia. The control group was selected from agematched healthy female candidates with no history of platelet disorders or medications that may affect platelet function.

All samples were collected according to hospital guidelines and after obtaining informed consent in accordance with approved specimen collection protocols. Consent was given by 102 people for participation. The protocol was approved by the Ethics Committee of Liaocheng People's Hospital (number 2012126).

2.2. Clinical Characteristics. All patients were registered in the hospital admission system. Information regarding age, gender, surgical resection, tumor histology, hormonal receptor evaluation, and so forth could be accessed and collected from this system. Breast cancer staging was performed after surgery by histology and pathology according to the 2009 American Joint Committee Cancer Staging Manual. Hormonal receptors for estrogen (ER) and progesterone (PR) were regularly quantified by immunohistochemistry at the Department of Pathology, Liaocheng People's Hospital, following histology evaluation for breast cancer.

2.3. Sample Collection and Processing. Platelet isolation from whole blood was performed as described by Bergers et al. [21]. In brief, human venous blood $(4 \mathrm{~mL})$ was drawn into two tubes precoated with sodium citrate. Platelet-rich plasma was isolated by centrifugation of whole blood at $170 \mathrm{~g}$ for $15 \mathrm{~min}$ and then at $900 \mathrm{~g}$ for $10 \mathrm{~min}$. PP were precipitated as white pellets and supernatants were retained as PPP samples. All samples were stored at $-80^{\circ} \mathrm{C}$.

Platelet pellet lysis was performed by using Abcam platelet isolation methods (isolation of human platelets from whole blood, Abcam). Lysis buffer (2x) consisted of 2\% NP40, $30 \mathrm{mM}$ Hepes, $150 \mathrm{mM} \mathrm{NaCl}$, and $2 \mathrm{mM}$ EDTA, at $\mathrm{pH} 7.4$.
Lysis buffer $(50 \mu \mathrm{L})$ was added to each pellet sample and vigorously mixed by pipetting and vortexing. PBS was used to dilute the lysed samples.

2.4. Measurement of VEGF, PDGF, TSP-1, PF4, TGF- $\beta 1$, and Actin. Angiogenesis regulators were measured in PP and PPP. Patients with platelet disorders or a medication history that would affect platelet physiology were excluded from the study. All proteins were assessed by commercial ELISA kits according to manufacturer's protocols \{human PDGF (HG04176), human TSP-1 (HG02106), human PF4 (HG021006), human actin (HG00954), human VEGF (HG06076), and human TGF- $\beta 1$ (HG00468), IBL, Germany\}. Preliminary experiments were performed to determine the optimal dilution range for each marker.

Activation of latent TGF- $\beta 1$ was performed by acidifying with $\mathrm{HCl}$ and neutralizing with $\mathrm{NaOH}$ [22]. Briefly, each $100 \mu \mathrm{L}$ sample was added to precoated wells and incubated for $1-2 \mathrm{~h}$ at $37^{\circ} \mathrm{C}$ as described in the kit protocols. After washing, $100 \mu \mathrm{L}$ of conjugated secondary antibody was added and incubated for $1 \mathrm{~h}$ at $37^{\circ} \mathrm{C}$ or room temperature. The plates were washed three times; the substrate was added and incubated for $30 \mathrm{~min}$ at $37^{\circ} \mathrm{C}$, followed by addition of the detection substrate and incubation for $25 \mathrm{~min}$ at room temperature. After a final wash, TMB substrate $(100 \mu \mathrm{L})$ was added, and the mixture was incubated at $37^{\circ} \mathrm{C}$ for $30 \mathrm{~min}$. The colorimetric reaction was stopped after $30 \mathrm{~min}$ with $100 \mu \mathrm{L}$ stop solution. The results were analyzed by a Thermo Scientific microplate spectrophotometer at $450 \mathrm{~nm}$.

2.5. Normalization of Protein Concentration and Quality Control. Actin levels were used to normalize the platelet contents in each sample. Protein contents in PP samples were also normalized to actin, thus eliminating potential bias introduced during processing and lysis. By associating platelet actin levels with platelet counts, we verified our method of PP lysis. A reference PP sample (platelet control), made from PPP samples, was used to monitor platelet lysis, dilution, and testing as a common reference for conversion from platelet counts to platelet protein level [23]. All standards and samples were run in duplicate and results were averaged.

2.6. Statistical Analysis. Median levels and interquartile ranges of each protein in breast cancer patients $(n=37)$ were compared to those of healthy controls $(n=65)$ using the nonparametric Mann-Whitney $U$ test. Protein contents in PP were normalized and expressed per $10^{6}$ platelets; the results are shown in box-and-whisker plots [24]. Receiver operating characteristic (ROC) and area under the curve (AUC) with the Youden index were used to identify optimal cut-off values and evaluate the ability of each biomarker to differentiate cancer patients from controls [25]. Patients with platelet disorders or a medication history that would affect platelet physiology were excluded from the study. Pearson $\chi^{2}$ test or Fisher's exact test was applied to identify differences in protein levels between patient groups. The Statistical Package for Social Sciences v. 16.0 was used for all statistical analyses and significance was defined as $P<0.05$. 
TABLE 1: Biomarker levels in PP and clinical characteristics.

\begin{tabular}{|c|c|c|c|c|c|}
\hline Groups & Number of patients & PF4 (ng) & TGF- $\beta$ (ng) & VEGF (pg) & PDGF-BB (pg) \\
\hline \multicolumn{6}{|l|}{ Stage } \\
\hline $\mathrm{I}+\mathrm{II}$ & 24 & $21.6 \pm 11.3$ & $16.5 \pm 10.6^{\mathrm{a}}$ & $2.37 \pm 1.0^{\mathrm{a}}$ & $40.4 \pm 13.3^{\mathrm{a}}$ \\
\hline $\mathrm{III}+\mathrm{IV}$ & 13 & $24.4 \pm 11.5$ & $24.6 \pm 12.2$ & $3.38 \pm 1.28$ & $49.9 \pm 13.8$ \\
\hline \multicolumn{6}{|l|}{ Age } \\
\hline$>60$ & 8 & $22.9 \pm 11.8$ & $23.1 \pm 12.5$ & $2.84 \pm 1.12$ & $50.3 \pm 14.6$ \\
\hline $40-60$ & 23 & $22.3 \pm 11.6$ & $20.16 \pm 17.4$ & $3.05 \pm 1.23$ & $46.3 \pm 13.5$ \\
\hline$<40$ & 6 & $23.3 \pm 12.9$ & $20.8 \pm 18.0$ & $2.89 \pm 1.11$ & $44.6 \pm 13.1$ \\
\hline \multicolumn{6}{|l|}{ LN status } \\
\hline No & 11 & $22.1 \pm 11.2$ & $16.4 \pm 10.1^{\mathrm{b}}$ & $2.32 \pm 1.05^{\mathrm{b}}$ & $40.2 \pm 13.1^{\mathrm{b}}$ \\
\hline N13 & 26 & $22.8 \pm 12.1$ & $24.5 \pm 11.3$ & $3.31 \pm 1.14$ & $49.8 \pm 13.5$ \\
\hline \multicolumn{6}{|c|}{ Molecular subtypes } \\
\hline Luminal A & 9 & $21.9 \pm 11.7$ & $18.4 \pm 11.6^{\mathrm{c}}$ & $2.66 \pm 1.09^{c}$ & $42.8 \pm 13.4^{\mathrm{c}}$ \\
\hline Luminal B & 12 & $22.1 \pm 12.3$ & $19.7 \pm 12.9$ & $2.73 \pm 1.17$ & $43.2 \pm 13.3$ \\
\hline HER-2(+) & 7 & $24.9 \pm 12.9$ & $28.9 \pm 13.7$ & $3.51 \pm 1.25$ & $52.1 \pm 14.3$ \\
\hline Basal like & 9 & $22.2 \pm 11.1$ & $33.2 \pm 15.2$ & $3.74 \pm 1.26$ & $56.2 \pm 14.7$ \\
\hline
\end{tabular}

Variables are normalized to actin and expressed per $10^{6}$ platelets. Being constrained by relatively low number of candidates in each clinical subgroup, we used average content instead of median level to make the comparisons. ${ }^{a}$ Stages I+II versus III+IV: $P<0.05,{ }^{\mathrm{b}} \mathrm{LN}$ status N0 versus N1-3: $P<0.05$, and ${ }^{\mathrm{c}}$ Luminal A or B versus HER-2 or Basal like: $P<0.05$.

TABLE 2: Levels of biomarkers in PP of the cancer and control study groups.

\begin{tabular}{lccccc}
\hline \multicolumn{3}{c}{$\begin{array}{c}\text { Cancer group } \\
(n=37)\end{array}$} & \multicolumn{2}{c}{$\begin{array}{c}\text { Control group } \\
(n=65)\end{array}$} \\
\hline Pariable & Median & Range & Median & Range \\
PF4, ng & 42.9 & $13.5-116.4$ & 19.1 & $9.3-48.9$ & $<0.001^{*}$ \\
TSP-1, ng & 21.2 & $3.1-63.2$ & 10.2 & $4.2-20.5$ & $<0.001^{*}$ \\
TGF- $\beta$, ng & 29.2 & $15.4-123.9$ & 27.0 & $11.5-120.5$ & 0.821 \\
VEGF, pg & 15.3 & $0.4-56.0$ & 4.3 & $0.5-16.9$ & $<0.001^{*}$ \\
\hline
\end{tabular}

Variables are normalized to actin and expressed per $10^{6}$ platelets.

${ }^{*}$ Statistically significant.

\section{Results}

3.1. Clinical Characteristics. The cancer patients represented four cancer stages and were aged 26 to 68 years. Platelet counts for all patients were within the normal range (105 295 $\left.\times 10^{9} / \mathrm{L}\right)$. Clinical characteristics and ER/PR results are listed in Table 1.

3.2. Normalization to Platelet Count. Actin concentrations were determined by ELISA and used to assess variations in PP volume after sample preparation. Actin quantities were similar between samples, demonstrating a close correlation between actin content and platelet count (Figure 1); however, we used the actin level in pooled platelets as a reference for protein normalization. Concentrations of all test proteins were calculated based on actin content and normalized to the actin level per $10^{6}$ platelets.

3.3. Platelet Sequestration of Angiogenesis Regulatory Proteins in Breast Cancer. PP and PPP from breast cancer patients and healthy controls were assessed. Compared with controls

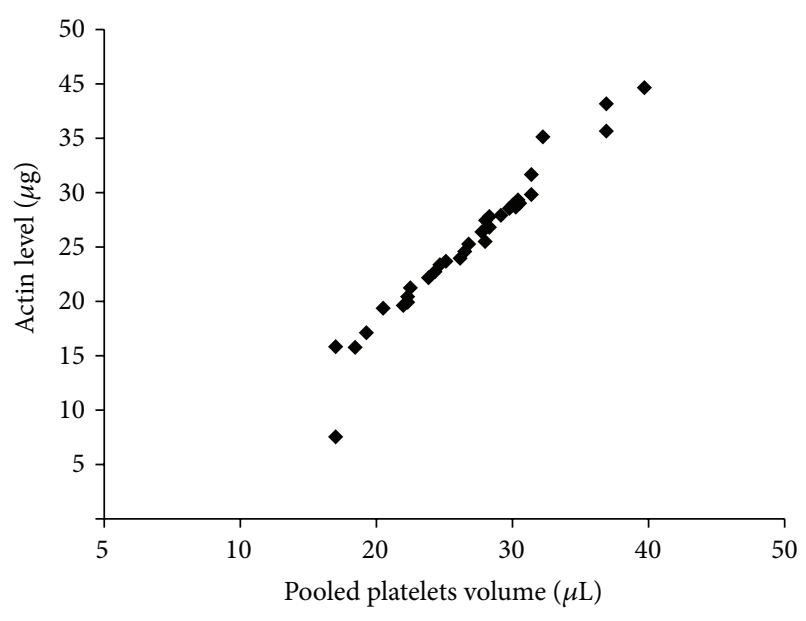

FIGURE 1: Dot plot of actin content and platelet count.

group, the median levels of VEGF, PF4, and PDGF-BB were significantly increased in PP from cancer patients (Table 2). Box-and-whisker plots for the cancer and control groups 


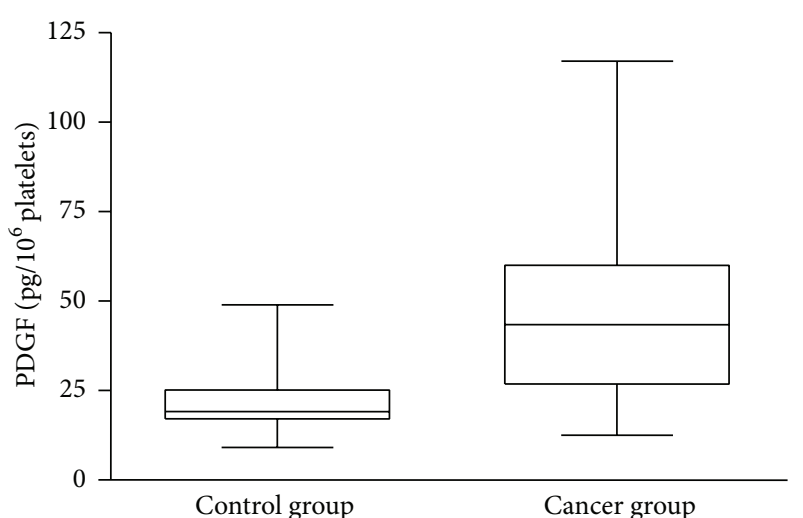

(a)

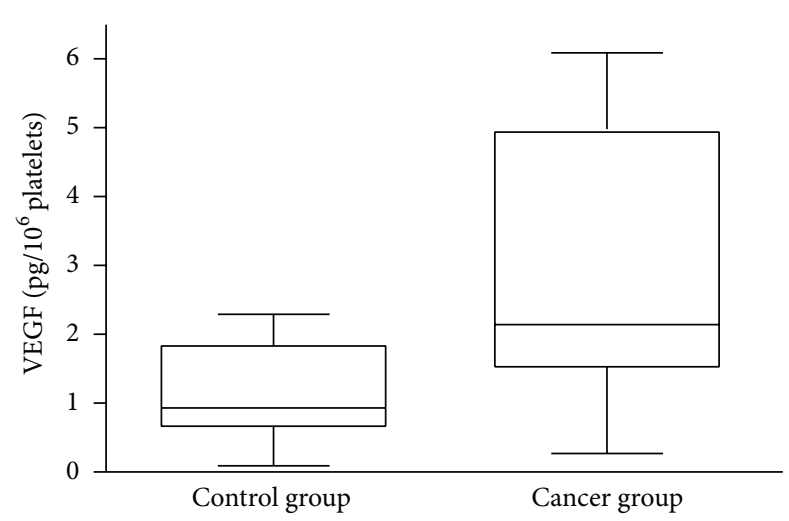

(c)

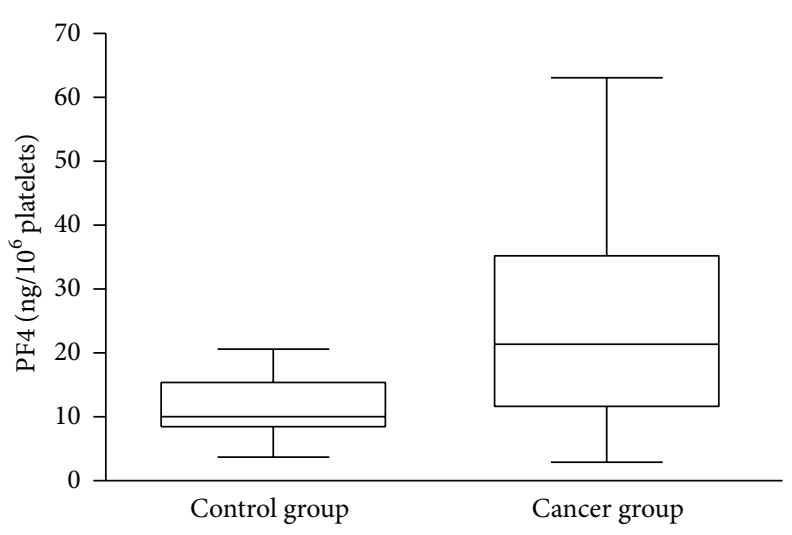

(b)

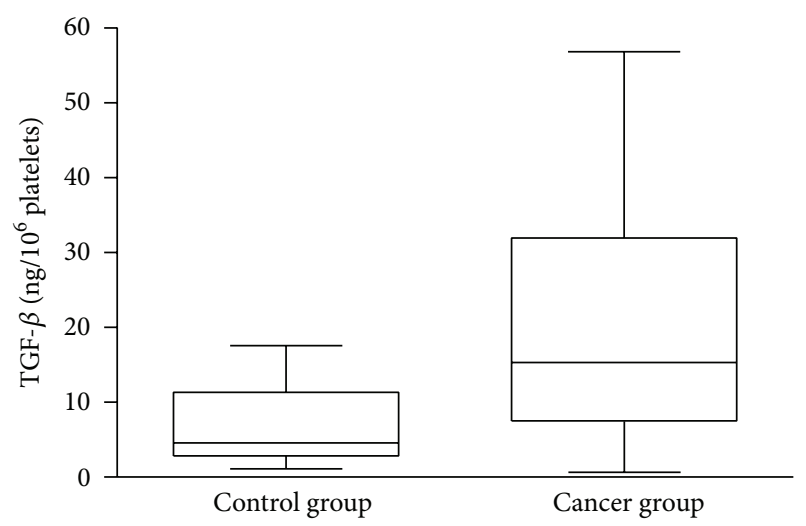

(d)

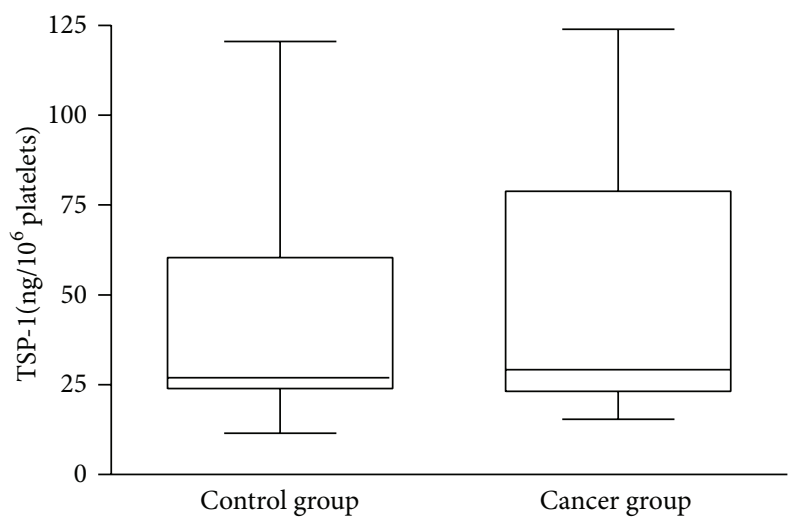

(e)

Figure 2: Box plot of PDGF, PF4, VEGF, TSP-1, and TGF- $\beta$ in PP. The box indicates the lower and upper quartile and the central line is the median. The points at the ends of the whiskers are the 2.5 and $97.5 \%$ values. PDGF (a), PF4 (b), VEGF (c), and TGF- $\beta$ (d).

for four of the five biomarkers in PP illustrate elevated levels of PDGF-BB (Figure 2(a)), PF4 (Figure 2(b)), VEGF (Figure 2(c)), and TGF- $\beta$ (Figure 2(d)). We also measured VEGF, PF4, PDGF-BB, and TGF- $\beta$ in PPP samples. With the exception of TGF- $\beta$, there were no significant differences between the cancer and control groups (Table 3 ).

3.4. TGF- $\beta 1$ in PP and PPP Samples from Cancer Patients. TGF- $\beta 1$ is significantly elevated in cancer patients compared with healthy controls. Compared to the control group, the level of TGF- $\beta 1$ in lysed PP was much higher $\left(15.3 \mathrm{ng} / 10^{6}\right.$ platelets versus $4.3 \mathrm{ng} / 10^{6}$ platelets, $\left.P<0.001\right)$. However, in the PPP samples, TGF- $\beta 1$ was lower in the cancer group than in the healthy controls; this maybe possibly due to sequestration of TGF- $\beta 1$ by circulating platelets ( 24.2 versus $42.48 \mathrm{ng} / \mathrm{mL}, P<0.001)$.

The level of PDGF (AUC: 0.856, 95\% CI: 0.771-0.940), PF4 (AUC: 0.735, 95\% CI: 0.612-0.859), VEGF (AUC: 0.789, 95\% CI: 0.694-0.884), and TGF- $\beta 1$ (AUC: 0.763 , 95\% CI: $0.658-0.868)$ in PP is significantly different between the cancer groups and control groups (Table 4). The ROC curve indicates the optimal cut-off points for each platelet biomarker 
TABLE 3: Biomarker levels in PPP.

\begin{tabular}{lcccccc}
\hline & \multicolumn{3}{c}{$\begin{array}{c}\text { Cancer group } \\
\text { Biomarker }\end{array}$} & Units & \multicolumn{2}{c}{ Control group } \\
& & Mean & SD & Mean & SD & \\
\hline PDGF & $\mathrm{pg} / \mathrm{mL}$ & 236.9 & 79.6 & 263.2 & 63.1 & 0.076 \\
PF4 & $\mathrm{ng} / \mathrm{mL}$ & 269.3 & 83.8 & 302.4 & 85.0 & 0.077 \\
TSP-1 & $\mathrm{ng} / \mathrm{mL}$ & 418.8 & 101.6 & 395.9 & 102.6 & 0.448 \\
VEGF & $\mathrm{pg} / \mathrm{mL}$ & 53.8 & 16.1 & 53.3 & 11.1 & 0.669 \\
TGF- $\beta$ & $\mathrm{ng} / \mathrm{mL}$ & 24.2 & 5.9 & 42.48 & 10.0 & $<0.001^{*}$ \\
\hline
\end{tabular}

Variables are not normalized.

* Statistically significant.

(Table 5). Combined accuracy for all four biomarkers as a set yielded excellent discrimination between cancer and healthy controls in PP $(P<0.0001)$ (Figure 3$)$.

\subsection{Angiogenesis Protein and Epithelial-Mesenchymal Tran-} sition Factors Were Correlated with Clinical Characteristics. To investigate the correlation between clinical characteristics and protein accumulation in platelets, we divided the breast cancer patients into groups according to age $(<40$ versus $40-60$ versus $>60$ ), cancer stage (stages I+II versus stages III+IV), hormonal receptor expression (low versus medium versus high). We found that the level of VEGF, PDGF-BB, and TGF- $\beta 1$ in platelet was associated with clinical characteristics (Table 1).

\section{Discussion}

Numerous reports have suggested the activation role of platelets in cancer progression, tumor growth, and metastasis [26-28]. There are a large number of angiogenic proteins in platelets, including VEGF, PDGF, TGF- $\beta 1$, angiopoietin-1 (Ang-1), and basic fibroblast growth factor (bFGF). These factors can promote vascular endothelial cell proliferation and formation of new blood vessels, which provide a suitable microenvironment for implantation and growth of tumor cells [29].

Recent study has found that the level of angiogenesis protein was increased in serum and tumor cells in patients with cancer [30-32]. Angiogenesis is a prognostic indicator for a variety of tumors [33]. However, the levels of many angiogenic proteins in plasma or serum can be detected until tumor load becomes large enough [34]. Angiogenesis markers quantification in breast cancer and their correlation with many clinicopathological prognostic variable angiogenic proteins are present in much higher concentrations in the platelets of experimental animals with malignant tumors than in the plasma, serum, and nonmalignant tumors [19]. Thus, regulation protein in platelets is more sensitive than that of them in plasma [35].

Our current data indicated that VEGF, PF4, PDGF-BB, and TGF- $\beta 1$ levels were elevated in PP of the cancer group but not in PPP. These were consistent with the previous study of McDowell et al. [36], and we expanded the number of breast cancer patients on the basis. For detection of these

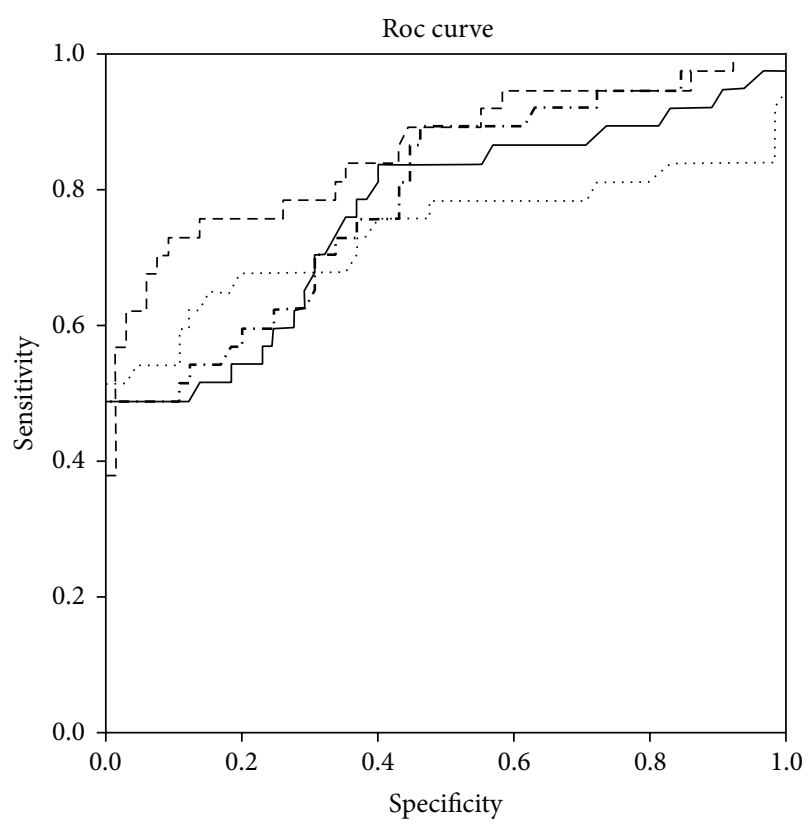

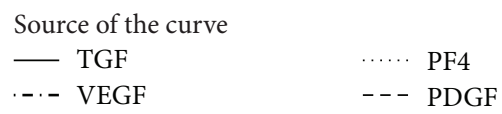

FIGURE 3: Receiver operating characteristic (ROC) curves of PDGF, PF4, VEGF, and TGF- $\beta$ in PP. Dashed 45-degree line is the reference. ROC curves are based on continuous variables for each biomarker normalized to actin and expressed per $10^{6}$ platelets.

proteins, we found that the curve of ROC was more than 0.7 , indicating that these proteins exhibit high sensitivity and specificity. They seem to be potential candidates for breast cancer diagnosis.

Zaslavsky et al. demonstrated that the expression of TSP-1 in platelet is increased in a mouse model with Lewis lung cancer cell lines [37], suggesting that TSP-1 is an inhibitor of tumor angiogenesis in the early stages of tumor growth. However, we found that there is no significantly different of TSP1 expression between the cancer groups and control groups. The reason for this phenomenon maybe is the difference in tumor burden between animals and human patients. However, the sample size is small and the results must be interpreted with caution.

In the present study, TGF- $\beta 1$ sequestration was increased in PP but decreased in PPP, although changes of concentration in PP are more robust. This may be due to elevated sequestration of TGF- $\beta 1$ by platelets in cancer patients.

Angiogenesis factors are strongly correlated with the prognosis and clinical pathology, especially for breast cancer [38-40]. VEGF expression is higher in breast cancer tissue and is closely associated with ER labeling index, lymph node metastasis, clinical stage, and histological grade [41]. We are also trying to understand whether the concentrations of vascular regulatory proteins in platelets are also linked to cancer status. All four stages of breast cancer samples were studied. We found that the level of VEGF, PDGF, and TGF- $\beta 1$ was associated with lymph node metastasis, clinical stage, 
TABLE 4: ROC curves of biomarkers of PP in the cancer group.

\begin{tabular}{|c|c|c|c|c|c|}
\hline \multirow{2}{*}{ Biomarker } & \multirow{2}{*}{ AUC } & \multirow{2}{*}{ Standard error } & \multirow{2}{*}{$P$ value } & \multicolumn{2}{|c|}{$95 \% \mathrm{CI}$} \\
\hline & & & & Lower limit & Upper limit \\
\hline PDGF & 0.856 & 0.043 & 0.001 & 0.771 & 0.940 \\
\hline TGF- $\beta$ & 0.763 & 0.054 & 0.001 & 0.658 & 0.868 \\
\hline VEGF & 0.789 & 0.048 & 0.001 & 0.694 & 0.884 \\
\hline PF4 & 0.735 & 0.063 & 0.001 & 0.612 & 0.859 \\
\hline
\end{tabular}

All $P$ values were less than 0.05 .

TABLE 5: Optimal cut-off point of biomarkers of PP in the cancer group.

\begin{tabular}{lcccc}
\hline Biomarker & AUC & $\begin{array}{c}\text { Optimal } \\
\text { cut-off }\end{array}$ & Sensitivity (\%) & Specificity (\%) \\
\hline PDGF pg & 0.856 & 26.65 & 75.7 & 86.2 \\
TGF- $\beta$ ng & 0.763 & 7.55 & 70.5 & 69.2 \\
VEGF pg & 0.789 & 1.655 & 70.3 & 69.2 \\
PF4 ng & 0.735 & 15.3 & 67.6 & 80.0 \\
\hline
\end{tabular}

Variables are normalized to actin and expressed per $10^{6}$ platelets.

and molecular subtype. Molecular portraits of human breast tumors are usually divided into four subtypes: luminal A, luminal B, HER-2(+), and basal-like [42]. Luminal A is usually associated with better prognosis, but HER-2(+) and basal-like subtypes indicate poor prognosis [43]. The result reported here reveals that the level of VEGF, PDGF, and TGF$\beta 1$ was higher than the latter two subtypes. This result is consistent with previous findings that ER-, PR-negative breast cancer has a high microvascular transfer rate and blood transfer risk $[44,45]$. The correlation between these markers and the clinical characteristics of cancer may play an important role in breast cancer diagnosis and prognosis. Compared with tissue immunohistochemistry, ELISA method is simpler and less invasive than immunohistochemistry and is easy to perform.

In conclusion, we found that circulating platelets in breast cancer sequester higher levels of PF4, VEGF, PDGF-BB, and TGF- $\beta 1$, and these proteins can be possible indicators for breast cancer in early diagnosis. The level of VEGF, PDGF, and TGF- $\beta 1$ expression may be associated with cancer prognosis. Further analysis of protein expression in platelets before and after cancer treatment (surgery, chemotherapy, or radiotherapy) is needed to evaluate their utility as markers for disease monitoring.

\section{Conflict of Interests}

The authors declare that there is no conflict of interests regarding the publication of this paper.

\section{Acknowledgment}

The study was supported by Key Technologies R \& D Program of Shandong province (Grant no. 2012GSF11845).

\section{References}

[1] R. Siegel, D. Naishadham, and A. Jemal, "Cancer statistics, 2013," CA: Cancer Journal for Clinicians, vol. 63, no. 1, pp. 11-30, 2013.

[2] J. Folkman, "What is the evidence that tumors are angiogenesis dependent?" Journal of the National Cancer Institute, vol. 82, no. 1, pp. 4-6, 1990.

[3] B. Janic and A. S. Arbab, "The role and therapeutic potential of endothelial progenitor cells in tumor neovascularization," TheScientificWorldJournal, vol. 10, pp. 1088-1099, 2010.

[4] E. Fuhrmann-Benzakein, M. N. Ma, L. Rubbia-Brandt et al., "Elevated levels of angiogenic cytokines in the plasma of cancer patients," International Journal of Cancer, vol. 85, no. 1, pp. 4045, 2000.

[5] P. Carmeliet, "Angiogenesis in life, disease and medicine," Nature, vol. 438, no. 7070, pp. 932-936, 2005.

[6] J. Folkman, "Angiogenesis: an organizing principle for drug discovery?” Nature Reviews Drug Discovery, vol. 6, no. 4, pp. 273-286, 2007.

[7] C. Dosquet, M.-C. Coudert, E. Lepage, J. Cabane, and F. Richard, "Are angiogenic factors, cytokines, and soluble adhesion molecules prognostic factors in patients with renal cell carcinoma?" Clinical Cancer Research, vol. 3, part 1, no. 12, pp. 24512458, 1997.

[8] B. Abendstein, G. Daxenbichler, G. Windbichler et al., "Predictive value of uPA, PAI-1, HER-2 and VEGF in the serum of ovarian cancer patients," Anticancer Research, vol. 20, no. 1, pp. 569-572, 2000.

[9] J. Kisucka, C. E. Butterfield, D. G. Duda et al., "Platelets and platelet adhesion support angiogenesis while preventing excessive hemorrhage," Proceedings of the National Academy of Sciences of the United States of America, vol. 103, no. 4, pp. 855860, 2006.

[10] B. Ho-Tin-Noé, T. Goerge, S. M. Cifuni, D. Duerschmied, and D. D. Wagner, "Platelet granule secretion continuously prevents intratumor hemorrhage," Cancer Research, vol. 68, no. 16, pp. 6851-6858, 2008.

[11] R. J. K. Hettiarachchi, S. M. Smorenburg, J. Ginsberg, M. Levine, M. H. Prins, and H. R. Buller, "Do heparins do more than just treat thrombosis? The influence of heparins on cancer spread," Thrombosis and Haemostasis, vol. 82, no. 2, pp. 947-952, 1999.

[12] M. H. Einstein, E. A. Pritts, and E. M. Hartenbach, "Venous thromboembolism prevention in gynecologic cancer surgery: a systematic review," Gynecologic Oncology, vol. 105, no. 3, pp. 813-819, 2007.

[13] L. Borly, P. Wille-Jørgensen, and M. S. Rasmussen, "Systematic review of thromboprophylaxis in colorectal surgery-an update," Colorectal Disease, vol. 7, no. 2, pp. 122-127, 2005.

[14] S. M. Smorenburg, R. J. K. Hettiarachchi, R. Vink, and H. R. Büller, "The effects of unfractionated heparin on survival in 
patients with malignancy-a systematic review," Thrombosis and Haemostasis, vol. 82, no. 6, pp. 1600-1604, 1999.

[15] R. Möhle, D. Green, M. A. S. Moore, R. L. Nachman, and S. Rafii, "Constitutive production and thrombin-induced release of vascular endothelial growth factor by human megakaryocytes and platelets," Proceedings of the National Academy of Sciences of the United States of America, vol. 94, no. 2, pp. 663-668, 1997.

[16] C. H. Heldin, B. Westermark, and A. Wasteson, "Plateletderived growth factor. Isolation by a large-scale procedure and analysis of subunit composition.”, Biochemical Journal, vol. 193, no. 3, pp. 907-913, 1981.

[17] E. Cenni, D. Granchi, M. Vancini, and A. Pizzoferrato, "Platelet release of transforming growth factor- $\beta$ and $\beta$-thromboglobulin after in vitro contact with acrylic bone cements," Biomaterials, vol. 23, no. 6, pp. 1479-1484, 2002.

[18] G. Klement, L. Kikuchi, M. Kieran, N. Almog, T.-T. Yip, and J. Folkman, "Early tumor detection using platelet uptake of angiogenesis regulators," Blood, vol. 104, no. 11, p. 839, 2004.

[19] G. L. Klement, T.-T. Yip, F. Cassiola et al., "Platelets actively sequester angiogenesis regulators," Blood, vol. 113, no. 12, pp. 2835-2842, 2009.

[20] R. Salgado, I. Benoy, J. Bogers et al., "Platelets and vascular endothelial growth factor (VEGF): a morphological and functional study," Angiogenesis, vol. 4, no. 1, pp. 37-43, 2001.

[21] G. Bergers, S. Song, N. Meyer-Morse, E. Bergsland, and D. Hanahan, "Benefits of targeting both pericytes and endothelial cells in the tumor vasculature with kinase inhibitors," Journal of Clinical Investigation, vol. 111, no. 9, pp. 1287-1295, 2003.

[22] D. J. Grainger, D. E. Mosedale, J. C. Metcalfe, P. L. Weissberg, and P. R. Kemp, "Active and acid-activatable TGF- $\beta$ in human sera, platelets and plasma," Clinica Chimica Acta, vol. 235, no. 1, pp. 11-31, 1995.

[23] J. E. Peterson, D. Zurakowski, J. E. Italiano Jr. et al., "Normal ranges of angiogenesis regulatory proteins in human platelets," American Journal of Hematology, vol. 85, no. 7, pp. 487-493, 2010.

[24] D. G. Altman, Practical Statistics for Medical Research, CRC Press, New York, NY, USA, 1990.

[25] M. S. Pepe, The Statistical Evaluation of Medical Tests for Classification and Prediction, vol. 28 of Oxford Statistical Science Series, Oxford University Press, Oxford, UK, 2003.

[26] M. Labelle, S. Begum, and R. Hynes, "Direct signaling between platelets and cancer cells induces an epithelial-mesenchymallike transition and promotes metastasis," Cancer Cell, vol. 20, no. 5, pp. 576-590, 2011.

[27] R. L. Stone, A. M. Nick, I. A. McNeish et al., "Paraneoplastic thrombocytosis in ovarian cancer," The New England Journal of Medicine, vol. 366, no. 7, pp. 610-618, 2012.

[28] E. Camerer, A. A. Qazi, D. N. Duong, I. Cornelissen, R. Advincula, and S. R. Coughlin, "Platelets, protease-activated receptors, and fibrinogen in hematogenous metastasis," Blood, vol. 104, no. 2, pp. 397-401, 2004.

[29] E. Sierko and M. Z. Wojtukiewicz, "Platelets and angiogenesis in malignancy," Seminars in Thrombosis and Hemostasis, vol. 30, no. 1, pp. 95-108, 2004.

[30] T. Szarvas, T. Jäger, F. Droste et al., "Serum levels of angiogenic factors and their prognostic relevance in bladder cancer," Pathology and Oncology Research, vol. 15, no. 2, pp. 193-201, 2009.

[31] F. Tas, D. Duranyildiz, H. Oguz, H. Camlica, V. Yasasever, and E. Topuz, "Circulating serum levels of angiogenic factors and vascular endothelial growth factor receptors 1 and 2 in melanoma patients," Melanoma Research, vol. 16, no. 5, pp. 405411, 2006.

[32] O. Balacescu, I. Neagoe, L. Balacescu et al., "Angiogenesis serum protein quantification for prostate pathology," Current Urology, vol. 2, no. 4, pp. 181-187, 2009.

[33] C. Benazzi, A. Al-Dissi, C. Chau et al., "Angiogenesis in spontaneous tumors and implications for comparative tumor biology," The Scientific World Journal, vol. 2014, Article ID 919570, 16 pages, 2014.

[34] D. Cervi, T.-T. Yip, N. Bhattacharya et al., "Platelet-associated PF-4 as a biomarker of early tumor growth," Blood, vol. 111, no. 3, pp. 1201-1207, 2008.

[35] H.-P. Gerber, A. K. Malik, G. P. Solar et al., "VEGF regulates haematopoietic stem cell survival by an internal autocrine loop mechanism," Nature, vol. 417, no. 6892, pp. 954-958, 2002.

[36] G. McDowell, I. Temple, C. Li et al., "Alteration in platelet function in patients with early breast cancer," Anticancer Research, vol. 25, no. 6 B, pp. 3963-3966, 2005.

[37] A. Zaslavsky, K.-H. Baek, R. C. Lynch et al., "Platelet-derived thrombospondin-1 is a critical negative regulator and potential biomarker of angiogenesis," Blood, vol. 115, no. 22, pp. 46054613, 2010.

[38] A. Balsari, J. A. M. Maier, M. I. Colnaghi, and S. Ménard, "Correlation between tumor vascularity, vascular endothelial growth factor production by tumor cells, serum vascular endothelial growth factor levels, and serum angiogenic activity in patients with breast carcinoma," Laboratory Investigation, vol. 79, no. 7, pp. 897-902, 1999.

[39] J. Zhao, F. Yan, H. Ju, J. Tang, and J. Qin, "Correlation between serum vascular endothelial growth factor and endostatin levels in patients with breast cancer," Cancer Letters, vol. 204, no. 1, pp. 87-95, 2004.

[40] D. Duranyildiz, H. Camlica, H. O. Soydinc, D. Derin, and V. Yasasever, "Serum levels of angiogenic factors in early breast cancer remain close to normal," Breast, vol. 18, no. 1, pp. 26-29, 2009.

[41] J. Rykala, K. Przybylowska, I. Majsterek et al., "Angiogenesis markers quantification in breast cancer and their correlation with clinicopathological prognostic variables," Pathology and Oncology Research, vol. 17, no. 4, pp. 809-817, 2011.

[42] C. M. Perou, T. Sørile, M. B. Eisen et al., "Molecular portraits of human breast tumours," Nature, vol. 406, no. 6797, pp. 747-752, 2000.

[43] L. A. Carey, C. M. Perou, C. A. Livasy et al., "Race, breast cancer subtypes, and survival in the Carolina Breast Cancer Study," The Journal of the American Medical Association, vol. 295, no. 21, pp. 2492-2502, 2006.

[44] J. Adams, P. J. Carder, S. Downey et al., "Vascular endothelial growth factor (VEGF) in breast cancer: comparison of plasma, serum, and tissue VEGF and microvessel density and effects of tamoxifen," Cancer Research, vol. 60, no. 11, pp. 2898-2905, 2000.

[45] S. J. Kim, N. Ikeda, E. Shiba, Y. Takamura, and S. Noguchi, "Detection of breast cancer micrametastases in peripheral blood using immunomagnetic separation and immunocytochemistry," Breast Cancer, vol. 8, no. 1, pp. 63-69, 2001. 


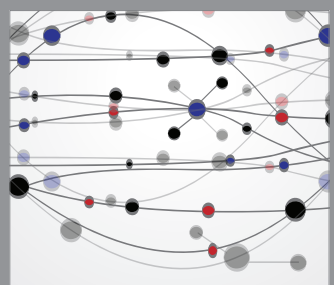

The Scientific World Journal
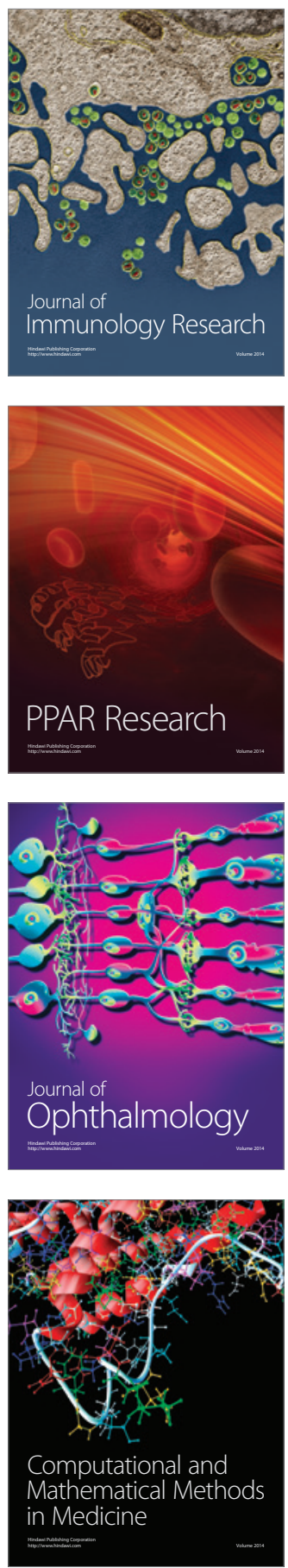

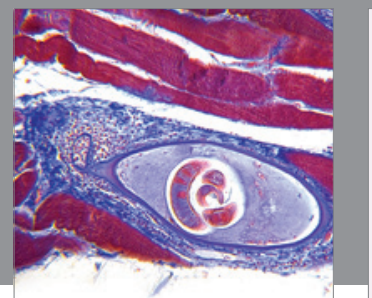

Gastroenterology

Research and Practice
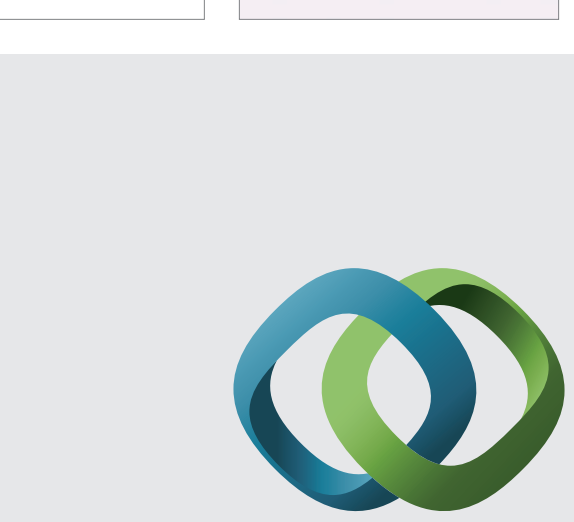

\section{Hindawi}

Submit your manuscripts at

http://www.hindawi.com
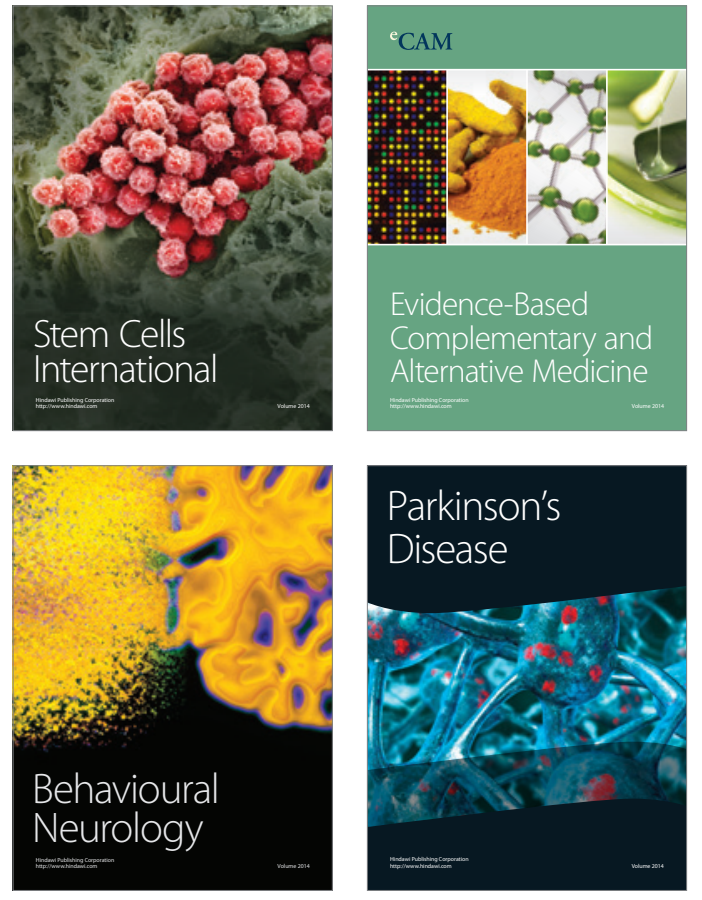
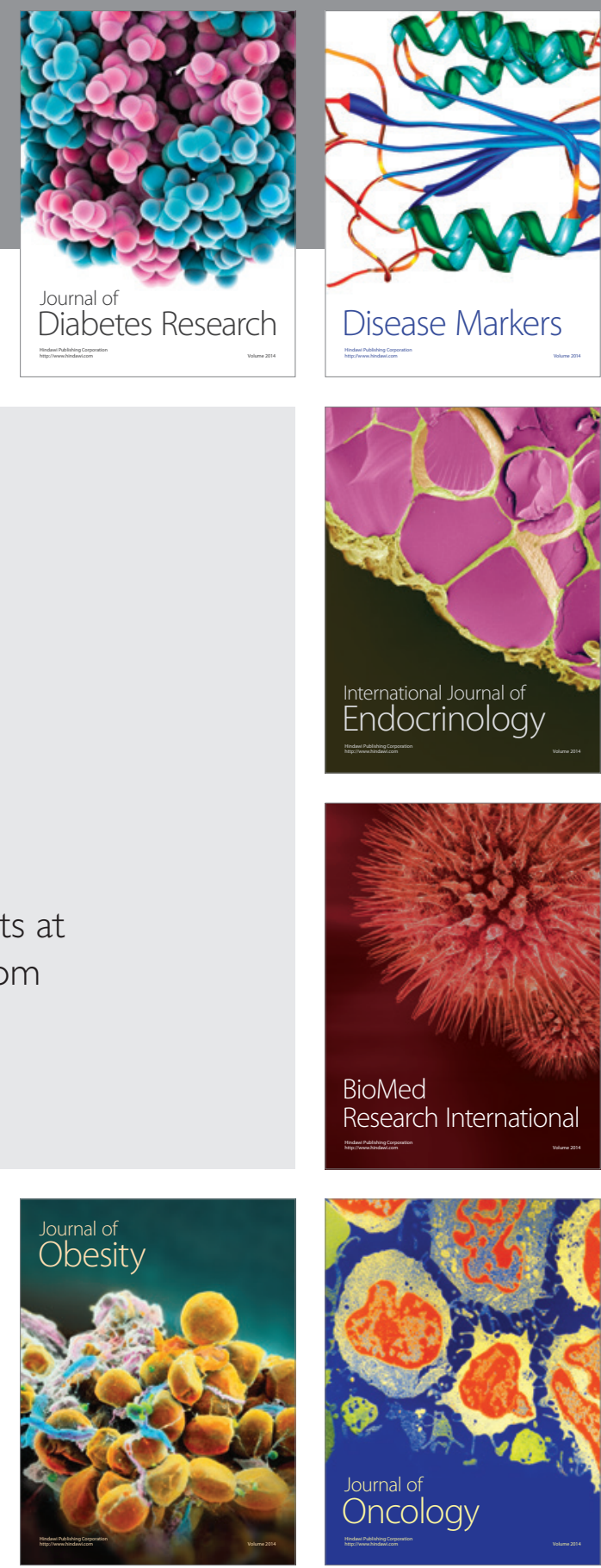

Disease Markers
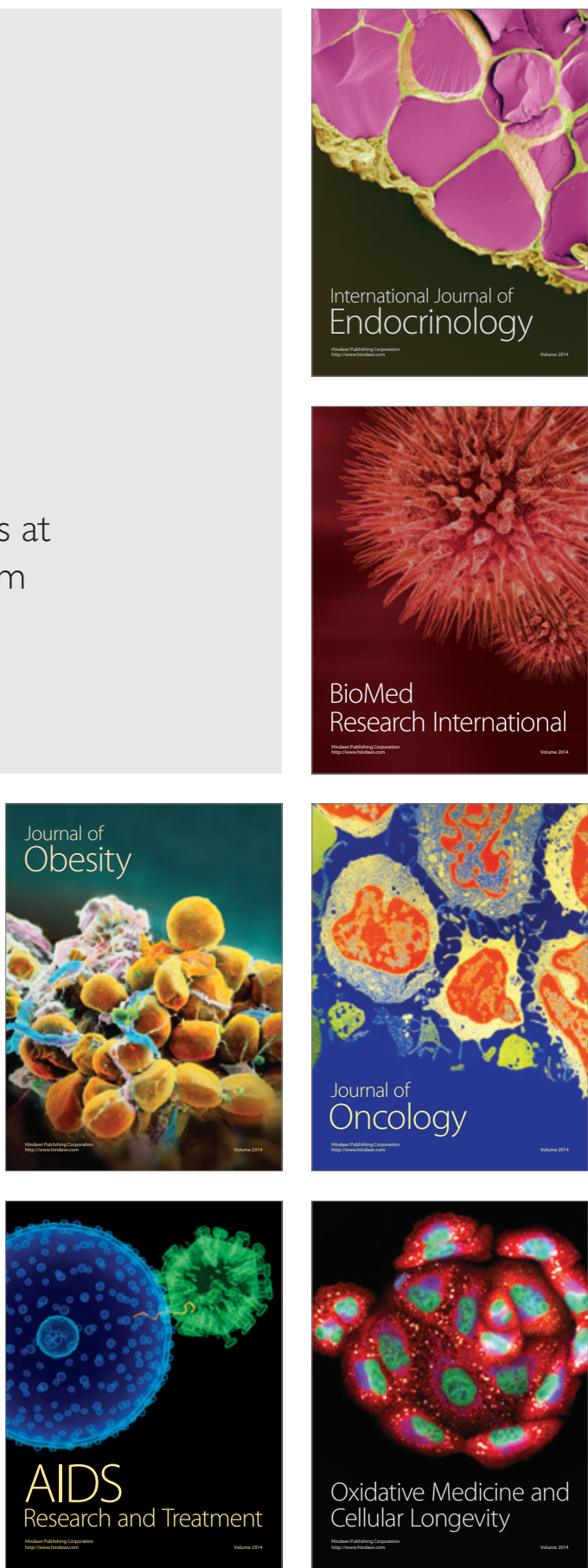